Pacific Journal of Mathematic 


\title{
ON KRULL OVERRINGS OF AN AFFINE RING
}

\section{WiLLIAM HEINZER}

\begin{abstract}
By an overring of an integral domain $A$ we mean a ring which contains $A$ and is contained in the quotient field of $A$. We consider the following question. If $D$ is a Krull overring of an affine ring $A$ is $D$ necessarily Noetherian? Our main result is an affirmative answer to this question when $A$ is a normal affine ring of dimension two defined over a field or pseudogeometric Dedekind domain such that each localization of $A$ has torsion class group.
\end{abstract}

We recall that an integral domain $J$ is called a Krull ring if $J$ is an intersection of rank one discrete valuation rings, say $J=\bigcap_{\alpha} V_{\alpha}$, such that each nonzero element of $J$ is a nonunit in only finitely many of the $V_{\alpha}$. One may assume that each $V_{\alpha}$ is an overring of $J$ and is irredundant in the representation $J=\bigcap_{\alpha} V_{\alpha}$. In this case each $V_{\alpha}$ is centered on a minimal prime (prime of height one) of $J$ and if $V_{\alpha}$ has center $P_{\alpha}$ on $J$, then $J_{P_{\alpha}}=V_{\alpha}$. The set $\left\{V_{\alpha}\right\}=\left\{J_{P_{\alpha}}\right\}$ is called the set of essential valuation rings for $J$. We use the notation $E(J)$ to denote the set of essential valuation rings of the Krull ring $J$.

A one dimensional Krull ring is a Dedekind domain and hence is Noetherian. There exist non-Noetherian 3 dimensional Krull rings, an example being given by Nagata [6, p. 207] who showed that the derived normal ring of a 3 dimensional local domain need not be Noetherian. Whether a 2 dimensional Krull ring is necessarily Noetherian remains open $^{1}$. Since the derived normal ring of a 2 dimensional Noetherian domain is again Noetherian one can not hope to construct non-Noetherian 2 dimensional Krull rings by a method similar to Nagata's. Our results here show that in certain special cases 2 dimensional Krull rings are Noetherian. In fact, the original motivation for our work was to determine if each Krull overring of $Z[X]$ ( $Z$ the ring of integers and $X$ an indeterminate over $Z$ ) is Noetherian, a question brought to our attention by Jack $\mathrm{Ohm}$. We are grateful to $\mathrm{Ohm}$ for several helpful conversations concerning this topic.

2. We will consistently use $A$ to denote a normal affine ring of dimension 2 defined over a field or pseudogeometric Dedekind domain. We will further assume that each localization $R$ of $A$ has torsion class

1 An exercise in Bourbaki [3, p. 83] outlines a method for constructing a two dimensional Krull ring which is asserted not to be Noetherian. However in [5] an argument is given to the effect that the Bourbaki construction must necessarily yield a Noetherian Krull ring. Recently Paul Eakin has constructed a non-Noetherian 2 dimensional Krull ring. 
group. This, of course, is equivalent to the assumption that each minimal prime of $R$ is the radical of a principal ideal.

Our first results concern Krull overrings of a localization of $A$. Let $R$ be a localization of $A$. $R$ has dimension either one or two and if $R$ has dimension one then $R$ is a rank one discrete valuation ring and has no nontrivial overrings. We assume therefore that $R$ is of dimension two with maximal ideal $M$. Let $D$ be a Krull overring of $R$. If $V$ is an essential valuation ring for $D$ then $V$ either has center $M$ on $R$ or else $V$ is centered on a minimal prime $P$ of $R$. In the latter case $R_{P} \subseteq V$, and since $R_{P}$ is also a rank one discrete valuation ring we have $R_{P}=V$ and $V \in E(R)$. Thus $E(D)-E(R)$ consists precisely of the essential valuation rings of $D$ having center $M$ on $R$ and the finiteness condition in the definition of a Krull ring insures that $E(D)-E(R)$ is a finite set.

If $V$ is a valuation overring of $R$ centered on $M$ we recall that the $R$-dimension of $V$ is defined to be the transcendence degree over $R / M$ of the residue field of $V$. (Here we are using the canonical embedding of $R / M$ in the residue field of $V$ ). Since $R$ is two dimensional and Noetherian each such $V$ has $R$-dimension either zero on one [1, p. 328]. Moreover, if $V$ has $R$-dimension zero then $V$ is necessarily centered on a maximal ideal of any domain between $R$ and $V$. Let $\left\{V_{i}\right\}_{i=1}^{n}$ be the subset of $E(D)-E(R)$ consisting of those elements of $E(D)-E(R)$ which have $R$-dimension zero and let $D^{\prime}$ be the Krull ring having $E(D)-\left\{V_{i}\right\}$ as its set of essential valuation rings. We now observe that to show $D$ is Noetherian it will suffice to show that $D^{\prime}$ is Noetherian. This is a consequence of the following proposition.

Proposition 1. Let $J$ be a Krull ring and let $V$ be an essential valuation ring for $J$ whose center $P$ on $J$ is a maximal ideal. Let $J^{\prime}$ be the Krull overring of $J$ having $E(J)-\{V\}$ as its set of essential valuation rings. If $J^{\prime}$ is Noetherian, then $J$ is Noetherian.

Proof. We note that $J^{\prime}$ is the $P$-transform of $J$ as defined by Nagata in $\left[7\right.$, p. 58]. Also $P J^{\prime} \cap J$ properly contains $P$ so that $P J^{\prime}=J^{\prime}$. Hence there is a one-to-one correspondence between the prime ideals of $J^{\prime}$ and the prime ideals of $J$ excluding $P$ where a prime ideal $Q^{\prime}$ of $J^{\prime}$ is associated with $Q^{\prime} \cap J=Q[7$, p. 58] or [8, p. 198]. We choose $\left\{x_{1}, \cdots, x_{n}\right\}=X \subseteq P$ so that $X J^{\prime}=J^{\prime}$. We may also assume that $X J_{P}=P J_{P}$. Then $X J=P$ since $X J_{Q}=P J_{Q}=J_{Q}$ for each maximal ideal $Q$ of $J$ distinct from $P$. Hence $P$ is finitely generated ${ }^{2}$. Let $Q$ be a prime of $J$ distinct from $P$ with $Q^{\prime}$ being the unique prime of

${ }^{2}$ We have in fact established that $P$ is invertible, for $P$ is finitely generated and localized at any maximal ideal $P$ is principal. 
$J^{\prime}$ such that $Q^{\prime} \cap J=Q$. By assumption $Q^{\prime}$ is finitely generated, say $\left\{y_{1}, \cdots, y_{m}\right\}=Y$ generates $Q^{\prime}$. There exists an integer $t$ such that $Y P^{t} \subseteq J$. Hence $Y J \cdot P^{t}=B$ is a finitely generated ideal of $J$ such that $B J^{\prime}=Q^{\prime}$. By enlarging $B$ if necessary we may assume that $B \not P$. Thus $B J_{P}=Q J_{P}=J_{P}$. If $N$ is a maximal ideal of $J$ distinct from $P$ and $N^{\prime}$ is the unique maximal ideal of $J^{\prime}$ with $N^{\prime} \cap J=N$ then $J_{N}=J^{\prime}{ }_{N^{\prime}}$. Hence $Q J_{N}=Q^{\prime} J_{N^{\prime}}=B J^{\prime}{ }_{N^{\prime}}$. It follows that $B=$ $Q[9$, p. 94]. We have thus shown that each prime ideal of $J$ is finitely generated and hence that $J$ is Noetherian. This completes the proof of Proposition 1.

We now construct a normal Noetherian ring $R^{\prime}$ such that $R^{\prime}$ is finitely generated over $R$ and $E\left(D^{\prime}\right) \subseteq E\left(R^{\prime}\right)$. Let $\left\{W_{i}\right\}_{i=1}^{m}=E\left(D^{\prime}\right)-E(R)$ and let $T_{i}$ be the maximal ideal of $W_{i}$. Since $W_{i}$ is a quotient ring of $D^{\prime}$ we see that $D^{\prime} / T_{i} \cap D^{\prime}$ has quotient field $W_{i} / T_{i}$. By assumption $W_{i} / T_{i}$ is transcendental over $R / T_{i} \cap R=R / M$. We choose $a_{i}$ in $D^{\prime}$ such that the residue of $a_{i}$ in $W_{i} / T_{i}$ is transcendental over $R / M$. Then $W_{i}$ is not centered on a maximal ideal of $R\left[a_{i}\right]$ so that $W_{i}$ is necessarily an essential valuation ring for $R^{\prime}$, the integral closure of $R\left[a_{1}, \cdots, a_{m}\right]$. Since $R^{\prime}$ is a finite $R\left[a_{1}, \cdots, a_{m}\right]$-module we conclude that $R^{\prime}$ is again a quotient ring of a normal affine ring of dimension two defined over a field or pseudogeometric Dedekind domain. Moreover $E\left(D^{\prime}\right) \subseteq E\left(R^{\prime}\right)$.

We proceed to show that $D^{\prime}$ is Noetherian. If $J$ is a Krull ring let $C(J)$ denote the class group of $J$ and let $C_{1}(J)$ be the torsion free quotient group $C(J) / C_{2}(J)$ where $C_{2}(J)$ is the torsion subgroup of $C(J)$. As Claborn observed in [4, p. 220] if $J$ and $J^{\prime}$ are Krull rings with $E\left(J^{\prime}\right) \subseteq E(J)$ then $C\left(J^{\prime}\right)$ is a homomorphic image of $C(J)$ and the kernel of this canonical homomorphism is generated by the classes of all minimal primes $P$ of $J$ such that $J_{P} \in E(J)-E\left(J^{\prime}\right)$. Since $C_{1}(R)$ is trivial $^{3}$ and $E\left(R^{\prime}\right)-E(R)$ is a finite set we see that $C_{1}\left(R^{\prime}\right)$ is finitely generated. Hence $C_{1}\left(R^{\prime}\right)$ is free abelian on a finite set of generators. The canonical homomorphism $\varphi: C\left(R^{\prime}\right) \rightarrow C\left(D^{\prime}\right)$ enduces an onto homomorphism $\varphi_{1}: C_{1}\left(R^{\prime}\right) \rightarrow C_{1}\left(D^{\prime}\right)$. Let $\left\{P_{i}\right\}_{i=1}^{k}$ be minimal primes of $R^{\prime}$ whose equivalence classes in $C_{1}\left(R^{\prime}\right)$ generate the kernel of $\varphi_{1}$. Let $Q=$ $\bigcap_{i=1}^{k} P_{i}$ and let $S$ be the $Q$-transform of $R^{\prime}$. Since $R^{\prime}$ is a quotient ring of a normal affine ring of absolute dimension two, Nagata's results in [7] and [8] imply that $S$ is finitely generated over $R^{\prime}$. Moreover the canonical homomorphism $\psi_{1}: C_{1}(S) \rightarrow C_{1}\left(D^{\prime}\right)$ is an isomorphism. This means that each minimal prime $P$ of $S$ such that $S_{P} \in E(S)-E\left(D^{\prime}\right)$ is the radical of a principal ideal which in turn implies that $D^{\prime}$ is a quotient ring of $S$. Since $S$ is Noetherian we conclude that $D^{\prime}$ is Noetherian ${ }^{4}$. We summarize the results of this section in the following theorem.

${ }^{3}$ It would suffice here to assume that $C_{1}(R)$ is finitely generated.

${ }^{4}$ We have actually shown that $D^{\prime}$ is a quotient ring of a normal affine ring. 
THEOREM 2. Let $R$ be a localization of a normal affine ring A, where $A$ is defined over a field on pseudogeometric Dedekind domain and has dimension two. If the class group of $R$ is a torsion group, or more generally if $C_{1}(R)$ is finitely generated, and if $D$ is a Krull overring of $R$ then $D$ is Noetherian.

3. We turn now to the consideration of an arbitrary Krull overring $D$ of $A$. Our main result is the following.

THEOREM 3. Let $A$ be a normal affine ring of dimension two defined over a field or psuedogeometric Dedekind domain and assume that each localization of $A$ has torsion class group. If $D$ is a Krull overring of $A$, then $D$ is Noetherian. ${ }^{5}$

Proof. Let $P^{\prime}$ be a prime ideal of $D$ and let $P=P^{\prime} \cap A$. If $S=A-P$ then $A_{S} \subseteq D_{S}$ and by Theorem $2 D_{S}$ is a Noetherian domain. Let $\mathrm{X}$ be a finite set of generators for $P$ and let $Y$ be a finite subset of $D$ such that $Y D_{S}=P^{\prime} D_{S}$. If $P$ is a maximal ideal of $A$ we observe that $X \cup Y=Z$ is a finite basis for $P^{\prime}$. For this purpose it will suffice to show that $Z D_{M^{\prime}}=P^{\prime} D_{M^{\prime}}$ for each maximal ideal $M^{\prime}$ of $D$. If $P \nsubseteq M^{\prime}$ then $X \nsubseteq M^{\prime}$ and $Z D_{M I^{\prime}}=P^{\prime} D_{M^{\prime}}=D_{M^{\prime}}$. However if $P \subseteq M^{\prime}$ then $D_{S} \subseteq D_{M^{\prime}}$. Hence $P^{\prime} D_{M^{\prime}}=Y D_{M^{\prime}}=Z D_{M^{\prime}}$. We conclude that $P^{\prime}$ is finitely generated when $P^{\prime} \cap A=P$ is a maximal ideal of $A$.

Consider now the case when $P$ is a minimal prime of $A$. We have $A_{P} \subseteq D_{P^{\prime}}$ and $A_{P}$ is a discrete rank one valuation ring. Hence $A_{P}=D_{P}$ and $D_{P}$ is an essential valuation ring for $D$. Now the nonzero elements of $P$ are positive in only finitely many of the essential valuation rings for $D$. Let $\left\{V_{i}\right\}_{i=1}^{n}$ be the essential valuation rings for $D$ distinct from $D_{P^{\prime}}$ in which the elements of $P$ are positive. (Of course the set $\left\{V_{i}\right\}$ may be empty). Each $V_{i}$ is centered on a maximal ideal $M_{i}$ of $A$. Let $S_{i}=A-M_{i}$. Then $A_{S_{i}} \subseteq D_{S_{i}}$ and again by Theorem 2, $D_{S_{i}}$ is a Noetherian domain. Let $Y_{i}$ be a finite subset of $D$ such that $Y_{i} D_{S_{i}}=P^{\prime} D_{S_{i}}$ and again let $X$ be a finite basis for $P$. In this case we set $Z=\bigcup_{i=1}^{n} Y_{i} \cup X$. If $M^{\prime}$ is a maximal ideal of $D$ and $P \nsubseteq M^{\prime}$ then as before $X \nsubseteq M^{\prime}$ and $Z D_{M^{\prime}}=P^{\prime} D_{M^{\prime}}=D_{M^{\prime}}$. If $P \subseteq M^{\prime}$ and $M^{\prime} \cap A=M_{i}$ then $Z D_{M M^{\prime}}=Y_{j} D_{M^{\prime}}=P^{\prime} D_{M H^{\prime}}$. In the remaining case let $M=M^{\prime} \cap A$ and $S=A-M$. We have $A_{S} \subseteq D_{S}$ and $E\left(D_{S}\right) \subseteq E\left(A_{S}\right)$. Moreover $C\left(A_{S}\right)$ is a torsion group so that $D_{S}$ is a quotient ring of $A_{S}\left[4\right.$, p. 219]. Hence $P^{\prime} D_{S}=P D_{S}=Z D_{S}$, and $P^{\prime} D_{M^{\prime}}=Z D_{M^{\prime}}$. We conclude that $P^{\prime}=Z D$ and hence that $D$ is Noetherian.

5 That not every Krull overring of a 3 dimensional normal affine ring need be Noetherian has recently been shown in joint work of the author and Paul Eakin. 
COROLlaRY 4. If $A$ is a polynomial ring in two variables over a field or more generally a polynomial ring in one variable over a pseudogeometric Dedekind domain, then each Krull overring of $A$ is Noetherian.

Proof. We need only observe that each localization of $A$ has torsion class group. If $A=D[X]$ with $D$ a Dedekind domain and if $P$ is a prime of height 2 in $A$ with $Q=P \cap D$ then $A_{P}$ is a quotient ring of the unique factorization domain $D_{Q}[X]$. Thus each localization of $A$ has torsion class group.

Added in proof. In a paper submitted to Proc. Amer. Math. Soc., the author has now shown that each Krull overring of a 2-dimensional Noetherian domain is again Noetherian.

\section{REFERENCES}

1. S. Abhyankar, On the valuations centered on a local domain, Amer. J. Math. 78 (1956), 321-348.

2. - Arithmetical algebraic geometry, Harper and Row, New York, 1965.

3. N. Bourbaki, Algebra commutative, Chapitre 7, Herman, Paris, 1965.

4. L. Claborn, Every abelian group is a class group, Pacific J. Math. 18 (1966), 219-222.

5. P. Eakin, and W. Heinzer, Some open questions on minimal primes of a Krull domain, Canad. J. Math. 20 (1968), 1261-1264.

6. M. Nagata, Local rings, Interscience, New York, 1962.

7. - A treatise on the $14^{\text {th }}$ problem of Hilbert, Mem. Coll. Sci. Univ. Kyoto, Ser.

A Math 30 (1956-57), 57-70. Addition and corrections, ibid 197-200.

8. - A theorem on finite generation of a ring, Nagoya Math. J. 27 (1966), 193205.

9. O. Zariski, and P. Samuel, Commutative algebra, Vol. II, D. Van Nostrand, Princeton, 1960.

Received February 26, 1968.

Louisiana State UnIVERsity 




\section{Pacific Journal of Mathematics \\ Vol. 29, No. 1 \\ May, 1969}

Jorge Alvarez de Araya, A Radon-Nikodým theorem for vector and operator

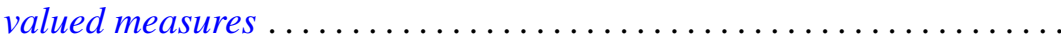

Deane Eugene Arganbright, The power-commutator structure of finite

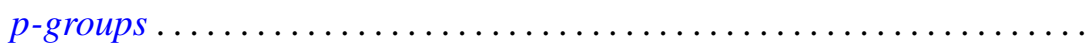

Richard Eugene Barlow, Albert W. Marshall and Frank Proschan, Some

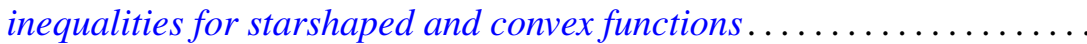

David Clarence Barnes, Some isoperimetric inequalities for the eigenvalues

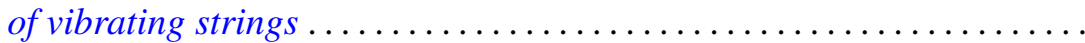

David Hilding Carlson, Critical points on rim-compact spaces ...........

Allan Matlock Weber Carstens, The lattice of pretopologies on an arbitrary

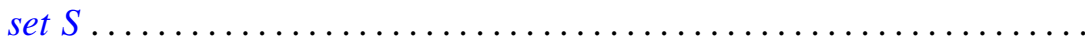

S. K. Chatterjea, A bilateral generating function for the ultraspherical

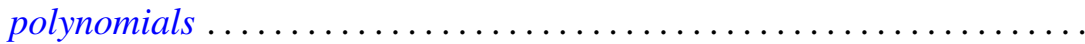

Ronald J. Ensey, Primary Abelian groups modulo finite groups ......... 77

Harley M. Flanders, Relations on minimal hypersurfaces ............ 83

Allen Roy Freedman, On asymptotic density in n-dimensions........... 95

Kent Ralph Fuller, On indecomposable injectives over artinian rings...... 115

George Isaac Glauberman, Normalizers of p-subgroups in finite groups . . . 137

William James Heinzer, On Krull overrings of an affine ring ........... 145

John McCormick Irwin and Takashi Ito, A quasi-decomposable abelian group without proper isomorphic quotient groups and proper isomorphic subgroups.

Allan Morton Krall, Boundary value problems with interior point boundary conditions

John S. Lowndes, Triple series equations involving Laguerre

polynomials

Philip Olin, Indefinability in the arithmetic isolic integers

Ki-Choul Oum, Bounds for the number of deficient values of entire functions whose zeros have angular densities..

R. D. Schafer, Standard algebras ....................

Wolfgang M. Schmidt, Irregularities of distribution. III.

Richard Alfred Tapia, An application of a Newton-like method to the Euler-Lagrange equation 